\title{
Classical Zeno dynamics in the light emitted by an extended, partially coherent source
}

\author{
Miguel A. Porras, ${ }^{1}$ Alfredo Luis, ${ }^{2}$ and Isabel Gonzalo ${ }^{2}$ \\ ${ }^{1}$ Departamento de Física Aplicada, Grupo de Sistemas Complejos, Universidad Politécnica de Madrid, Rios Rosas 21, 28003 Madrid, Spain \\ ${ }^{2}$ Departamento de Óptica, Facultad de Ciencias Físicas, Universidad Complutense, 28040 Madrid, Spain
}

(Received 4 September 2013; published 4 November 2013)

\begin{abstract}
We provide theoretical and experimental evidence of a classical-optics realization of quantum Zeno dynamics. In contrast to other approaches, the classical implementation is extremely simple and adaptable. Frequently monitoring the transverse spreading of the light emitted by an extended source results in a nontrivial dynamics where spreading and all other transverse effects associated with light propagation as the increase of spatial coherence tends to be inhibited. This is fully equivalent to a quantum Zeno dynamics where monitoring whether a quantum free particle at rest remains in a spatial interval causes inhibition of the quantum-state evolution, including the degree of purity.
\end{abstract}

DOI: 10.1103/PhysRevA.88.052101

PACS number(s): 03.65.Xp, 03.65.Ta, 42.25.Fx, 42.60.Da

\section{INTRODUCTION}

The Zeno effect was originally introduced as a quantum phenomenon in which observation inhibits the evolution of the observed system. Several versions were later discovered, of which the most advanced is the so-called Zeno dynamics [1], where observation does not necessarily inhibit evolution but places some constraints. Typically, observation forces the quantum state to remain within a given subspace of the system's Hilbert space, but otherwise, the system is free to evolve under some effective Hamiltonian tailored alike by the original free dynamics and the constraints caused by observation.

In this paper, we provide a classical-optics realization of quantum Zeno dynamics. Frequent monitoring the transversal spreading of the light emitted by an extended source results in a Zeno dynamics where transversal spreading and all other transversal effects usually associated with the propagation of light are inhibited. Despite its classical-optics nature, this is fully equivalent to a quantum Zeno dynamics of a free particle in which monitoring its presence within some prescribed spatial region tends to inhibit the evolution of the state. In sharp contrast to other examples of quantum Zeno dynamics currently in progress [2], this classical-optics approach has an extremely simple experimental implementation. This can be significant in order to investigate nontrivial examples of Zeno dynamics taking advantage of the simplicity that characterizes the classical-optics mimicking of quantum phenomena. For example, arbitrary transformations of the complex wave function can be implemented by liquid-crystal plates with easily tailored point-dependent complex transmittances. Different classical-optical counterparts of other Zeno versions can be found in Ref. [3].

The classical Zeno dynamics is rather counterintuitive to the common sense, even to experienced scientists. Prompted, if more or less of the light coming from an illuminated narrow slit (source) will fall on a distant identical slit (detector) when a number of identical aligned slits are inserted between them [Figs. 1(a) and 1(b)], almost all scientists we have asked respond "less light." Intuitively, diffraction spreading in each slit removes a fraction of the light power falling in the next slit. For coherent illumination, opticians familiarized with Fresnel zones know, however, that a few strategically placed intermediate slits can create a lens effect that increases the light power in the detector [4,5]. Recently, we have shown that, for coherent illumination, insertion of an equally spaced increasing number of slits always results in an increase of the light power on the detector for a large enough number of slits [6]. The same result holds irrespective of how the increasing numbers of slits are distributed, e.g., randomly, in the source-detector path so that it has no simple explanation in terms of Fresnel zones. Increasing power in the detector was interpreted as a (partial) inhibition of diffraction spreading caused by frequent diffraction.

The phenomenon is more striking and has deeper implications when the illumination is not spatially coherent, for example, sunlight. In the setup of Fig. 1 illuminated by partially coherent light, the power at the detector slit approaches its value at the source slit when a number of intermediate slits is inserted. The intermediate slits are found to be more efficient for increasing the power at the detector slit compared to the coherent case, which seems to contradict the fact that diffraction spreading of partially coherent light is more pronounced and, therefore, more difficult to halt. We then easily observe, experimentally, this effect in light power using standard light sources, a few slits, and a detector. Furthermore, common optical intuition suggests that successive truncation of the wave front in each slit will always result in higher coherence at the detector slit [7], but we actually find that the degree of coherence at the detector slit diminishes towards its value at the source when a number of intermediate slits is inserted (for a recent Zeno effect in coherence expressed as the degree of polarization, see Ref. [8]). More generally, we show theoretically that, in our particular case (observed free propagation), the state of light (defined by the mutual intensity $J$ in the quasimonochromatic case [9]) approaches its initial state at the source slit and, therefore, so does any observable property of light that might be expressed in terms of $J$.

This inhibition is a bona fide classical realization of a quantum Zeno dynamics. Fresnel diffraction is, indeed, formally equivalent to nonrelativistic evolution of a free particle with the replacement $k / z \rightarrow m / \hbar t$, where $k$ is the wave number, $z$ is the propagation distance, $m$ is the mass of the particle, and $t$ is time so that free-particle evolution may be referred to as diffraction in time [10]. In the quantum context, the density matrix $\rho$ in position representation replaces the mutual intensity $J$, light power becomes position probability, coherence becomes 

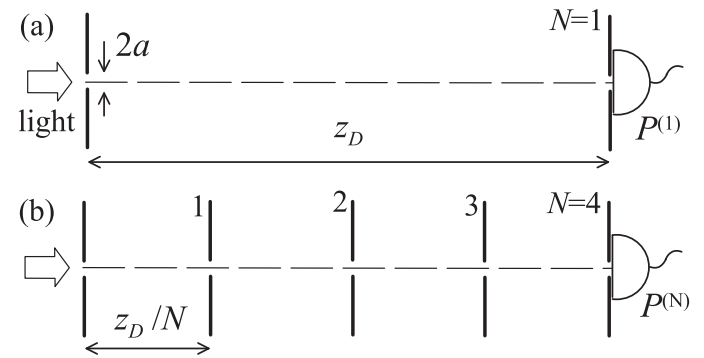

FIG. 1. Experimental setup for observation of Zeno dynamics in light propagation.

state purity, and the slits represent measurements of position, projecting the state on the spatial interval they define. Thus, the Zeno inhibition of transverse effects in light propagation in a distance $z_{D}$ is equivalent to a quantum Zeno inhibition of free-particle evolution in a time $t_{D}$ by repeated measurements of position. The detector reading of the light power that has crossed all slits is the classical version of the probability that the free particle has always been found within the slit regions. Actually, this is an extremely simple realization of the so-called dynamical Zeno effects since the particle can evolve freely within the subspace of positions defined by the slits [1].

\section{NUMERICAL EVALUATIONS OF LIGHT-POWER DYNAMICS}

In preliminary numerical simulations, we focus on quasimonochromatic partially coherent illumination with mutual intensity (within a scalar picture of light),

$$
J\left(x_{1}, x_{2}\right)=\left\langle E\left(x_{1}\right) E^{\star}\left(x_{2}\right)\right\rangle=\frac{1}{2 a} \exp \left[-\frac{\left(x_{1}-x_{2}\right)^{2}}{d^{2}}\right]
$$

for $x_{1}, x_{2} \in[-a, a]$ and zero otherwise. The intensity $I(x)=$ $J(x, x)$ within the slit is then uniform with transversal coherence length proportional to $d$. The factor $1 /(2 a)$ makes the power,

$$
P=\int_{-a}^{a} d x J(x, x)
$$

to be normalized to unity at the source slit. The degree of coherence between any pair of points in a slit is given by $\mu\left(x_{1}, x_{2}\right)=\left|J\left(x_{1}, x_{2}\right)\right| / \sqrt{I\left(x_{1}\right) I\left(x_{2}\right)}$ [9], and the global degree of coherence is characterized by [11]

$$
\mu_{g}=\frac{\left[\iint_{-a}^{a} d x_{1} d x_{2}\left|J\left(x_{1}, x_{2}\right)\right|^{2}\right]^{1 / 2}}{\int_{-a}^{a} d x J(x, x)}=\frac{\left[\operatorname{tr}\left(\rho^{2}\right)\right]^{1 / 2}}{\operatorname{tr} \rho},
$$

where the last equality is the quantum-mechanical counterpart in the form of quantum-state purity. In the Fresnel approximation of diffraction, the mutual intensity propagates according to

$$
\begin{aligned}
J\left(x_{1}^{\prime}, x_{2}^{\prime}\right)= & \frac{k}{2 \pi z} \iint_{-a}^{a} d x_{1} d x_{2} J\left(x_{1}, x_{2}\right) \\
& \times K\left(x_{1}^{\prime}-x_{1}\right) K^{\star}\left(x_{2}^{\prime}-x_{2}\right),
\end{aligned}
$$

where $K(x)=\exp \left(i k x^{2} / 2 z\right)$. With the slit labeling of Fig. 1, the mutual intensity $J^{(N)}\left(x_{1}, x_{2}\right)$ on the detector slit $N$ at the distance $z_{D}$ from the source after diffraction in $N-1$
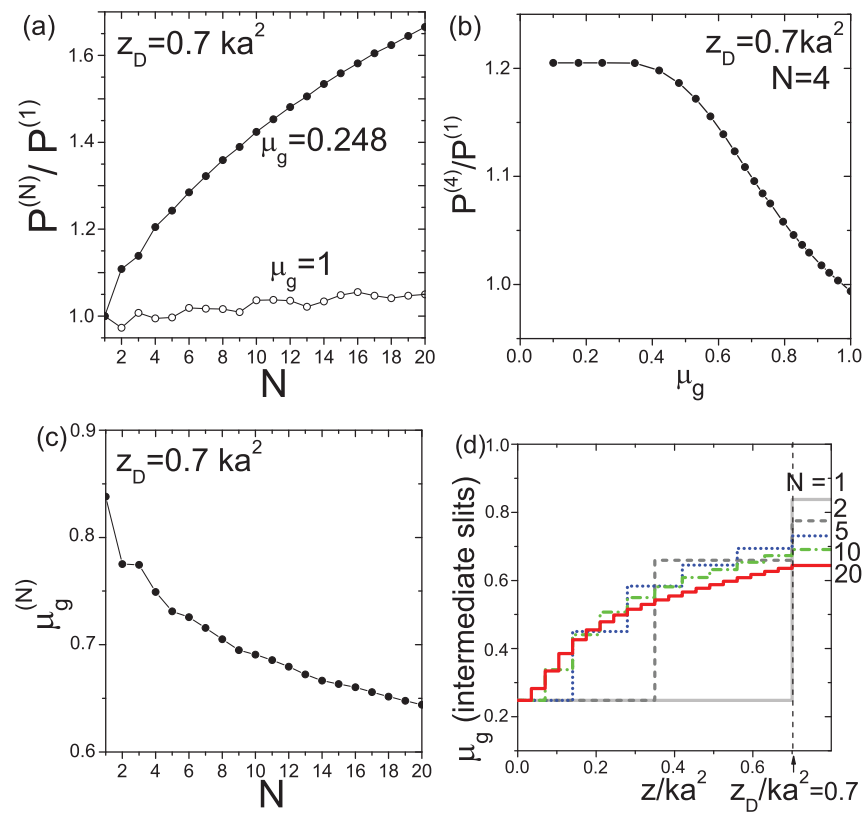

FIG. 2. (Color online) Numerical results: (a) Power gain $P^{(N)} / P^{(1)}$ on the detector slit as the number of intermediate slits $N-1$ increases for coherent illumination (open dots) and partially coherent illumination (dots). (b) Power gain $P^{(4)} / P^{(1)}$ for three intermediate slits as the degree of coherence of the source $\mu_{g}$ increases. (c) Global degree of coherence $\mu_{g}^{(N)}$ at the detector slit as the number of intermediate slits increases for partially coherent illumination with $\mu_{g}=0.248$. (d) Axial variation in the global degree of coherence from the source value $\mu_{g}=0.248$ to the detector value $\mu_{g}^{(N)}$ as light traverses the intermediate slits.

intermediate slits is obtained by cascaded application of Eq. (4) $N$ times with distances $z=z_{D} / N$.

Figure 2(a) shows the calculated ratios between the power $P^{(1)}$ on the detector without intermediate slits and the power $P^{(N)}$ with $N-1$ intermediate slits in the cases of coherent illumination with the plane-wave front $\left(\mu_{g}=1\right.$ for $\left.d=\infty\right)$ as in Ref. [6] and of partially coherent illumination $\left(\mu_{g}=\right.$ 0.248 for $d=0.1 a$ ) for a particular slit-detector distance $z_{D}$. Diffraction inhibition is evidenced by an increasing power on the detector as more and more intermediate slits are inserted compared to the case of no intermediate slits, i.e., $P^{(N)} / P^{(1)}$ is larger than unity and grows with $N$. For partially coherent illumination, the detected powers $P^{(1)}$ and $P^{(N)}$ are much smaller than with coherent illumination since diffraction spreading is faster, but the Zeno effect is more pronounced, i.e., the gain $P^{(N)} / P^{(1)}$ is considerably higher for any number of intermediate slits. In the example of Fig. 2(a), three of four slits suffice to obtain a gain of $120 \%$, whereas, coherent illumination requires tens of slits for a similar gain. For a particular source-detector distance $z_{D}$ and number of slits $N$, Fig. 2(b) illustrates the weakening of the Zeno effect as the degree of coherence of the source increases. Qualitatively similar results hold for other values of $z_{D}$ and $N$.

\section{OBSERVATION OF THE ZENO EFFECT IN LIGHT-POWER DYNAMICS}

We have experimentally confirmed the existence of this effect in light power using a light source, a few slits, and an 


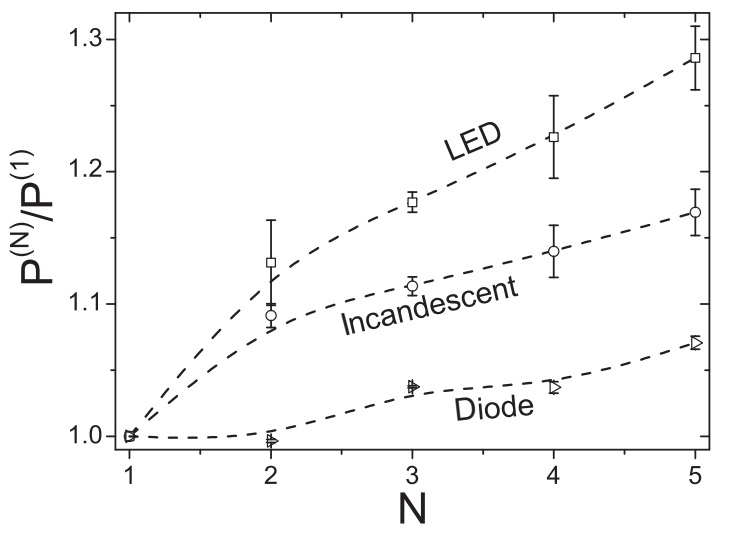

FIG. 3. Experimental results: For a commercial incandescent lamp, a white LED lamp, and a pigtailed laser diode $(\lambda=635 \mathrm{~nm})$ illuminating the system of slits of Fig. 1 with $a=0.3$ and $z_{D}=$ $500 \mathrm{~mm}$, measured power gain $P^{(N)} / P^{(1)}$ on the detector slit as a function of $N$. The symbols represent the average of a set of measurements, and the bars represent the standard deviations. The dashed curves are guides to the eye.

optical power meter in an optical bench. To show its generality, a commercial filament lamp with a frosted bulb, a commercial white light-emitting-diode (LED) lamp, and a pigtailed laser diode are used as sources. The width of the source, intermediate, and detector slits is $2 a=0.6 \mathrm{~mm}$. The filament and LED are placed as close as possible to the source slit or imaged by a lens on the source slit in order to increase the incoming light power, but the results are substantially the same in the two arrangements. The diverging output radiation from the fiber of the diode is collimated by a lens in a beam of cross section of about $1 \mathrm{~cm}$. The power meter is attached to the backface of the detector slit, placed at a distance of $z_{D}=500 \mathrm{~mm}$ from the source slit (about one diffraction length $k a^{2} / 2$ for the mean-visible wavelength), and collects all light falling on it. For each number $N-1$ of intermediate slits equally spaced $z_{D} / N$, we evaluate the gain $P^{(N)} / P^{(1)}$ at the detector slit as the quotient between power-meter indications with $N-1$ intermediate slits and with no intermediate slits. Alignment of the intermediate slits is achieved by carefully maximizing the power-meter indication each time that an intermediate slit is inserted. As seen in Fig. 3, the power gain $P^{(N)} / P^{(1)}$ on the detector slit is greater than unity and grows with the number of intermediate slits for all three sources. Despite the fact that the LED and filament lamps are not quasimonochromatic sources, the power gains are of the same order of magnitude as those obtained numerically from the Gaussian quasimonochromatic model. As expected, the Zeno effect is smaller and less monotonous for the more coherent laser diode.

\section{NUMERICAL EVALUATIONS OF COHERENCE DYNAMICS}

More counterintuitive is that the degree of coherence of the detector slit tends to retain its source value when intermediate slits are inserted. Spatial limitation by an opening of the radiation coming from a spatially incoherent source is well known to be the simplest way to obtain a more spatially coherent secondary source [7]. In Fig. 2(c), for example, the

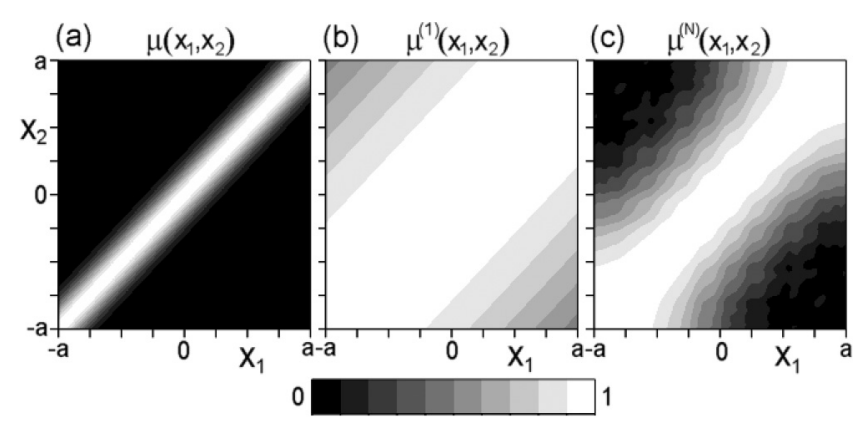

FIG. 4. Grayscale representation of the two-point degree of coherence $\mu\left(x_{1}, x_{2}\right)$, evaluated numerically, at (a) the source slit, (b) at the detector slit without intermediate $(N=1)$ slits, and (c) at the detector slit with 29 intermediate slits $(N=30)$. The source coherence length is $d=0.2 a\left(\mu_{g}=0.347\right)$, and the source-detector distance is $z_{D}=k a^{2}$.

global degree of coherence grows from $\mu_{g}=0.248$ to $\mu_{g}^{(1)}=$ 0.838 (coherence distances from $d=0.1 a$ to $d \simeq 1.7 a$ ) in free propagation from the source to the detector. One may also think that the enhanced inhibition of diffraction spreading in repeated diffraction is due to the increase in coherence in each intermediate slit that lowers diffraction spreading, but the numerical simulations show a more complex scenario. As seen in Fig. 2(c), the degree of coherence $\mu_{g}^{(N)}$ at the detector slit decreases towards the source value $\mu_{g}$ as more and more intermediate slits are inserted. The variation along $z$ of the global degree of coherence as light traverses the intermediate slits is shown in Fig. 2(d). Free-space propagation between two slits preserves the global degree of coherence (horizontal segments). Limitation of the propagating field by any of the intermediate slits suddenly raises the degree of coherence (vertical segments). However, the more the "steps up" in the global degree of coherence $(N=1,2,5,10,20)$, the lower the degree of coherence $\mu_{g}^{(N)}$ reached at the detector slit. The degree of coherence $\mu\left(x_{1}, x_{2}\right)$ between any pair of points at the detector also approaches its distribution at the source. For example, the short coherence length $(d=0.2 a)$ at the source [Fig. 4(a)] becomes much longer $(d \sim 1)$ at the detector slit upon free propagation without intermediate slits [Fig. 4(b)] and diminishes almost monotonically towards the source coherence length when more and more intermediate slits are placed [Fig. 4(c)].

\section{A SIMPLE THEORETICAL DESCRIPTION OF THE ZENO DYNAMICS}

Standard theoretical methods in optics allows us to prove that the slits or the position measurements inhibit the evolution of the state of light, defined by the mutual intensity $J\left(x_{1}, x_{2}\right)$ or of the particle state $\rho\left(x_{1}, x_{2}\right) \equiv\left\langle x_{1}|\rho| x_{2}\right\rangle=J\left(x_{1}, x_{2}\right)$ and, therefore, of all their properties. Passage of light through the system of slits in Fig. 1 is, indeed, equivalent to $N$ bounces of light in a resonator with finite-width planar strip mirrors $[7,12]$. The diffraction modes associated with the resonator are coherent fields that reproduce themselves from mirror to mirror (slit to slit) aside from a constant, i.e., $\psi_{m}(x) \rightarrow$ $\lambda_{m} \psi_{m}(x), m=1,2, \ldots, \infty$, where $\lambda_{m}$ is the mode eigenvalue [13]. Its squared modulus usually is written as $\left|\lambda_{m}\right|^{2}=1-\delta_{m}$, 
where $\delta_{1}<\delta_{2}<\delta_{3}$ are the mode losses per bounce. For planar strip mirrors spaced $L$ and if the resonator Fresnel number $N_{F}=k a^{2} /(2 \pi L)$ is greater than unity, the losses per bounce are given by $\delta_{m}=c_{m} N_{F}^{-3 / 2}$ with $c_{m} \simeq 0.118 m^{2}$ [14]. We assume that the mutual intensity of the partially coherent light on the source slit can suitably be represented as a sum of a number of uncorrelated diffraction modes [12],

$$
J\left(x_{1}, x_{2}\right)=\sum_{m} P_{m} \psi_{m}\left(x_{1}\right) \psi_{m}^{\star}\left(x_{2}\right),
$$

with adequate weights $P_{m}$. This representation allows writing the mutual intensity on the $N$ th slit after $N-1$ intermediate slits as $J^{(N)}\left(x_{1}, x_{2}\right)=\sum_{m}\left|\lambda_{m}\right|^{2 N} P_{m} \psi_{m}\left(x_{1}\right) \psi_{m}^{\star}\left(x_{2}\right)$ or

$$
J^{(N)}\left(x_{1}, x_{2}\right)=\sum_{m}\left[1-c_{m}\left(\frac{2 \pi L}{k a^{2}}\right)^{3 / 2}\right]^{N} P_{m} \psi_{m}\left(x_{1}\right) \psi_{m}^{\star}\left(x_{2}\right) \text {. }
$$

This expression has different limits at large $N$ depending on the meaning of $L$. For fixed $L$, all terms in the sum in (6) approach zero. The fundamental mode $m=1$ tends to zero slower compared to the other modes given its lower losses, which yields $J^{(N)}\left(x_{1}, x_{2}\right) \sim\left[1-c_{1}\left(2 \pi L / k a^{2}\right)^{3 / 2}\right]^{N} P_{1} \psi_{1}\left(x_{1}\right) \psi_{1}^{\star}\left(x_{2}\right)$ asymptotically at large $N$. This is the situation in an empty resonator as light travels back and forth repeatedly from mirror to mirror. The power tends to zero at the same time that the residual amount of light becomes coherent (the mutual intensity factorizes) due to repeated spatial filtering [7]. In the Zeno scheme, however, $L=z_{D} / N$. Use of the binomial theorem in Eq. (6) leads to

$$
\begin{aligned}
J^{(N)}\left(x_{1}, x_{2}\right)= & \sum_{m} P_{m} \psi_{m}\left(x_{1}\right) \psi_{m}^{\star}\left(x_{2}\right) \\
& \times\left[1-c_{m}\left(\frac{2 \pi z_{D}}{k a^{2}}\right)^{3 / 2} \frac{1}{N^{1 / 2}}+o\left(\frac{1}{N^{1 / 2}}\right)\right],
\end{aligned}
$$

which evidences that the mutual intensity at the detector approaches that at the source as the number of intermediate slits within $z_{D}$ increases. Similarly, the evolution of the density matrix tends to be inhibited as measurements of position within $t_{D}$ are more frequent.

As an example, Eq. (6) with only the fundamental and firstorder mode in Eqs. (2) and (3) gives, assuming that $\psi_{1}(x)$ and $\psi_{2}(x)$ are normalized to unity, $P^{(N)}=P_{1}\left|\lambda_{1}\right|^{2 N}+P_{2}\left|\lambda_{2}\right|^{2 N}$ and $\mu_{g}^{(N) 2}=\left(P_{1}^{2}\left|\lambda_{1}\right|^{4 N}+P_{2}^{2}\left|\lambda_{2}\right|^{4 N}\right) / P^{(N) 2}$. Note that evaluation of the degree of coherence from (3) for an arbitrary mixture of modes would require the knowledge of their shapes since the modes are not orthogonal, but the symmetry properties of the fundamental and first-order modes [13] make the above expression for $\mu_{g}^{(N)}$ independent of their shapes. Figure 5(a)
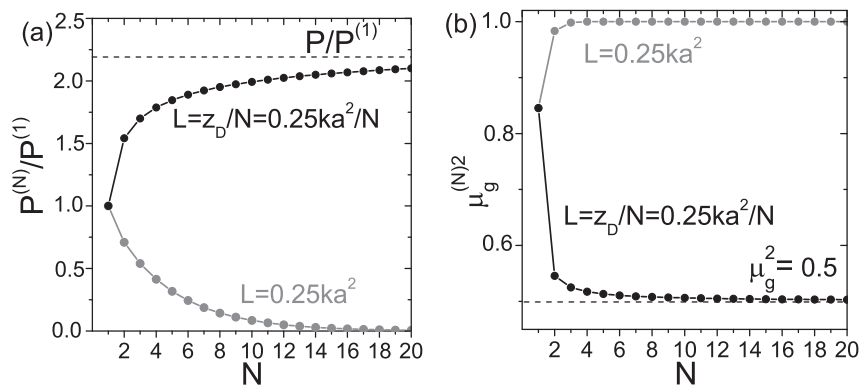

FIG. 5. From (6), (a) power gain and (b) global degree of coherence from as functions of $N$ for fixed distance $L=0.25 \mathrm{ka}^{2}$ (gray curves) and for $L=z_{D} / N$ with $z_{D}=0.25 \mathrm{ka}^{2}$ (black curves) in the Zeno scheme. The source is a mixture of the two modes with $P_{1}=P_{2}=0.5$ with degree of coherence $\mu_{g}^{2}=0.5$.

shows that the power $P^{(N)}$ tends to zero in the resonator arrangement (gray curve) but increases up to the source power in the Zeno scheme (black curve) following a similar trend as in the numerical simulations and in the measured values. Similarly, the coherence in the resonator is seen, in Fig. 5(b), to build up to unity (gray curve) but to decrease down to the source value in the Zeno arrangement (black curve).

\section{CONCLUSION}

Summarizing, we have presented classical and quantum Zeno dynamics that manifest preventing spreading and an increase in spatial coherence or state purity in the free evolution of a localized wave packet. To some extent, the coexistence of the two effects might be regarded as paradoxical since increasing power through a distant slit invokes collimation and spatial coherence, which is precisely prevented by the same scheme. Both effects actually are seen to coexist because the evolution of the state of light or of the quantum particle is inhibited. We also remark about the extremely simple experimental arrangement, accessible even to undergraduate laboratories, for the observation of this Zeno effect.

\section{ACKNOWLEDGMENTS}

M.A.P. acknowledges support from Projects No. MTM2012-39101-C02-01 and No. FIS2010-22082 of the Ministerio de Economía y Competitividad of Spain. A.L. acknowledges support from Projects No. FIS2012-35583 of the Spanish Ministerio de Economía y Competitividad and QUITEMAD No. S2009-ESP-1594 of the Consejería de Educación de la Comunidad de Madrid. I.G. acknowledges support from Project No. FIS2010-22082. We thank Dr. H. Canabal and Dr. F. Carreño for technical assistance.
[1] M. V. Berry and S. Klein, J. Mod. Opt. 43, 2139 (1996); M. V. Berry, J. Phys. A 29, 6617 (1996); P. Facchi and S. Pascazio, Phys. Rev. Lett. 89, 080401 (2002); J. Phys. A: Math. Theor. 41, 493001 (2008).
[2] J. M. Raimond, P. Facchi, B. Peaudecerf, S. Pascazio, C. Sayrin, I. Dotsenko, S. Gleyzes, M. Brune, and S. Haroche, Phys. Rev. A 86, 032120 (2012); F. Schaefer, I. Herrera, S. Cherukattil, C. Lovecchio, F. S. Cataliotti, F. Caruso, and A. Smerzi, QIPC 
2013: Quantum Information Processing and Communication (unpublished).

[3] M. Kitano, Opt. Commun. 141, 39 (1997); K. Yamane, M. Ito, and M. Kitano, ibid. 192, 299 (2001); A. Peres, Am. J. Phys. 48, 931 (1980); P. Kwiat, H. Weinfurter, T. Herzog, A. Zeilinger, and M. Kasevich, Ann. N. Y. Acad. Sci. 755, 383 (1995); S. Longhi, Phys. Rev. Lett. 97, 110402 (2006); P. Biagioni, G. Della Valle, M. Ornigotti, M. Finazzi, L. Duo, P. Laporta, and S. Longhi, Opt. Express 16, 3762 (2008).

[4] M. De, J. W. Y. Lit, and R. Tremblay, Appl. Opt. 7, 483 (1968).

[5] J. W. Y. Lit and R. Tremblay, J. Opt. Soc. Am. 59, 559 (1969); Opt. Commun. 1, 280 (1970).

[6] M. A. Porras, A. Luis, I. Gonzalo, and A. S. Sanz, Phys. Rev. A 84, 052109 (2011).
[7] E. Wolf, Phys. Lett. 3, 166 (1963).

[8] A. Luis, I. Gonzalo, and M. A. Porras, Phys. Rev. A 87, 064102 (2013).

[9] M. Born and E. Wolf, Principles of Optics, 7th expanded ed. (Cambridge University Press, Cambridge, UK, 1999).

[10] M. Moshinsky, Phys. Rev. 88, 625 (1952); E. Torrontegui, J. Muñoz, Y. Ban, and J. G. Muga, Phys. Rev. A 83, 043608 (2011).

[11] J. Tervo, T. Setälä, and A. T. Friberg, J. Opt. Soc. Am. A 21, 2205 (2004).

[12] W. Streifer, J. Opt. Soc. Am. 56, 1481 (1966).

[13] A. G. Fox and T. Li, Bell Syst. Tech. J. 40, 453 (1961).

[14] L. Ronchi, Appl. Opt. 9, 733 (1970). 